\title{
Escala de desenvolvimento auditivo e de linguagem na criança implantada
}

\author{
Hearing and language development scale in cochlear implanted \\ children
}

Claudia Andriguetto Maoski Moretti', Angela Ribas', Ana Cristina Guarinello', Marine Raquel Diniz da Rosa²

\begin{abstract}
RESUMO
Objetivo: Caracterizar o desempenho auditivo e de linguagem oral em crianças implantadas com menos de dois anos de idade auditiva, por meio da Escala de Desenvolvimento de Audição e Linguagem (EDAL-1) e comparar os resultados aos de crianças ouvintes até 2 anos de idade. Métodos: Estudo experimental de caráter descritivo. Participaram 141 crianças, divididas em dois grupos: grupo controle, com 92 normo-ouvintes, e grupo experimental, com 49 crianças surdas acompanhadas audiologicamente, durante os dois primeiros anos após a implantação. As normo-ouvintes passaram por avaliação auditiva e os pais responderam ao questionário EDAL-1. As crianças implantadas passaram por avaliação otorrinolaringológica e fonoaudiológica a cada três meses e os pais responderam ao EDAL-1. Resultados: Nos dois grupos foram observadas respostas ascendentes, com melhora de acordo com o aumento da idade cronológica. Os resultados obtidos no grupo experimental foram estatisticamente piores que no grupo controle, porém, com tendência de crescimento. O EDAL-1 mostrou-se de fácil aplicação e permitiu o monitoramento do desempenho das crianças implantadas. Conclusão: O desempenho auditivo e de linguagem oral em crianças surdas, usuárias de implante coclear, foi caracterizado pelo EDAL-1 e mostrou-se em crescimento, conforme o aumento do tempo de estimulação auditiva, fato também observado nas crianças ouvintes. Apesar disso, os resultados das crianças ouvintes foram melhores que os das crianças surdas implantadas.
\end{abstract}

Palavras-chave: Audição; Implante coclear; Linguagem; Protocolos clínicos

\begin{abstract}
Purpose: To characterize Auditory and oral language performance in children under 2 years of age with Hearing and Language Development Scale (EDAL-1) and to compare the results to those of hearing children up to two years of age. Methods: This is an experimental study of descriptive nature. A total of 141 children were divided into two groups: control group with 92 normal hearing, and the experimental group with 49 deaf children followed audiologically during the first two years after implantation. Normal hearing children underwent auditory assessment and parents responded to EDAL-1. The children implanted, every three months, underwent otorhinolaryngological and speech-language evaluation, and the parents answered EDAL-1. Results: In both groups we observed upward responses, with improvement as chronological and auditory age increases. Results obtained in the study group are statistically worse than in the control group, but with a tendency to grow. EDAL-1 was easy to apply and allowed to monitor the performance of the implanted children. Conclusion: Oral and auditory performance in deaf children with cochlear implants was characterized by EDAL-1, and it was shown to be growing as the time of auditory stimulation increased, a fact also seen in hearing children. Despite this, the results of the hearing children were better than those of the deaf children implanted.
\end{abstract}

Keywords: Hearing; Cochlear implantation; Language; Clinical protocols

\footnotetext{
Trabalho realizado no Programa de Pós-graduação em Distúrbios da Comunicação, Universidade Tuiuti do Paraná - UTP - Curitiba (PB), Brasil.

${ }^{1}$ Universidade Tuiuti do Paraná - UTP - Curitiba (PR), Brasil.

${ }^{2}$ Universidade Federal da Paraíba - UFPB - João Pessoa (PB), Brasil.

Conflito de interesses: Não.

Contribuição dos autores: CAMM responsável pela coleta e análise dos dados, revisão da literatura e elaboração do artigo; AR responsável pela análise dos dados e elaboração do artigo; ACG responsável pela revisão e elaboração do artigo; MRDR responsável pela revisão e elaboração do artigo.

Autor correspondente: Claudia Andriguetto Maoski Moretti. E-mail: Claudia.moretti@utp.br

Recebido: Julho 24, 2017; Aceito: Novembro 22, 2017
} 


\section{INTRODUÇÃO}

Os avanços tecnológicos têm marcado, de modo impressionante, os últimos anos da história da humanidade e, quando o tema é saúde auditiva, o grande marco é o implante coclear (IC). Há mais de 30 anos este dispositivo tem se mostrado eficiente e capaz de proporcionar acesso ao mundo sonoro às pessoas diagnosticadas com perda auditiva de grau severo e profundo.

O IC é um dispositivo eletrônico biomédico, biocompatível e durável, desenvolvido para realizar a função das células ciliadas danificadas ou não presentes na orelha interna. Modifica o sinal acústico e proporciona a estimulação elétrica das fibras remanescentes do nervo auditivo ${ }^{(1,2)}$, podendo levar a limiares auditivos praticamente normais.

Permitir a audição é a primeira meta do IC e, assim que o acesso ao mundo sonoro se estabelece, garantidos os limiares de audibilidade e de conforto acústico, espera-se que a linguagem oral se desenvolva ${ }^{(3)}$. Porém, apesar da sofisticação dos recursos eletrônicos alcançados atualmente, diversos fatores podem influenciar os resultados obtidos com o IC, como causa da perda auditiva, duração da surdez e, consequentemente, o tempo de privação sensorial, a idade do paciente ao receber o implante, a abordagem da reabilitação auditiva e de linguagem e a própria capacidade de neuroplasticidade do organismo ${ }^{(4)}$.

No Brasil, o IC é realidade no Sistema Único de Saúde (SUS) há mais de 20 anos e no estado do Paraná, mais especificamente na cidade de Curitiba, desde 2010 existem dois programas credenciados pelo SUS e capacitados para realizar o procedimento, sendo um deles em um hospital infantil, lócus deste estudo.

Neste serviço, em consonância com a Portaria GM/MS $\mathrm{n}^{\mathrm{o}} 2.776$ - 18 de dezembro de $2014^{(5)}$, os pacientes surdos são avaliados por equipe interdisciplinar e, se forem candidatos, são submetidos ao implante. Na sequência, o serviço deve prestar assistência vitalícia no que se refere às consultas de acompanhamento, para ativação e mapeamento do IC, bem como monitoração dos resultados.

No hospital infantil, de novembro de 2010 até junho de 2016, 65 crianças foram implantadas, sendo que 40\% eram oriundas de Curitiba, capital do estado onde está sediado o serviço, e $60 \%$ de outras cidades do estado do Paraná (litoral ou interior). Em função disso, a maioria das crianças faz terapia fonoaudiológica voltada para reabilitação de audição e linguagem, no município de origem ${ }^{(6)}$. Avaliar e monitorar o desempenho destas crianças é imprescindível para que orientações aos pais e terapeutas sejam encaminhadas satisfatoriamente.

No início dos trabalhos no serviço de saúde auditiva, em meados de 2010, a equipe de fonoaudiólogos utilizava instrumentos internacionais para registrar e fornecer informações sobre o desenvolvimento de audição e de linguagem oral dos pacientes implantados. Porém, percebeu-se a necessidade de elaborar um protocolo que atendesse às demandas regionais, ou seja, de fácil e rápida aplicação e de fácil compreensão por pais/responsáveis pelos pacientes. Foi nesse contexto que surgiu a Escala Brasileira de Desenvolvimento de Audição e Linguagem (EDAL) $)^{(7)}$, um protocolo rápido, composto por quatro testes, cada qual destinado à avaliação de crianças em determinadas faixas etárias.

O EDAL 1, primeiro teste da bateria destina-se à avaliação da evolução das habilidades auditivas e de linguagem oral, após a protetização.

O objetivo desta pesquisa foi verificar o desempenho auditivo e de linguagem oral em crianças implantadas com menos de dois anos de idade auditiva, por meio do EDAL-1 e comparar os resultados aos de crianças ouvintes até 2 anos de idade.

\section{MÉTODOS}

Trata-se de um estudo experimental, de caráter descritivo, aprovado pelo Comitê de Ética em Pesquisa da Universidade Tuiuti do Paraná (UTP), sob o número CEP 1.761.002/2016. Todos os responsáveis pelos participantes assinaram o Termo de Consentimento Livre e Esclarecido, autorizando a utilização dos dados coletados.

Foram avaliadas 141 crianças, cujos pais foram convidados a participar da pesquisa, divididas em dois grupos:

Grupo controle (GC): formado por 92 crianças normo-ouvintes recrutadas em sala de espera de serviço pediátrico credenciado pelo SUS, em Curitiba. Foram incluídas apenas crianças de 0 a 2 anos de idade cronológica, sem queixa de perda auditiva. Este grupo realizou avaliação otorrinolaringológica, audiometria comportamental em cabine acústica e imitanciometria. Os pais responderam ao EDAL-1 uma vez, logo após a consulta clínica.

Grupo experimental (GE): formado por 49 crianças surdas e implantadas. Foram incluídas crianças com perda auditiva severa ou profunda bilateral, usuárias de IC unilateral, com idade auditiva de zero a dois anos, que faziam controle trimestral e mapeamento do IC no serviço. Foram excluídas crianças com comorbidades capazes de interferir no desenvolvimento de linguagem. Este grupo realizou avaliação otorrinolaringológica, audiometria comportamental em cabine acústica e imitanciometria. Os pais ou responsáveis responderam ao EDAL-1, em cada sessão de balanceamento dos eletrodos.

Tanto o GC, como o GE, foram selecionados em serviço pediátrico credenciado pelo SUS, portanto, não houve preocupação em parear as crianças por nível social. Quanto à questão acadêmica, não foi possível fazer pareamento, pois a idade cronológica das crianças, entre os grupos, foi bastante heterogênea.

Sabe-se que a comparação da idade auditiva em uma criança implantada após os 3 anos e 5 meses é injusta, pois o tempo de privação auditiva não favorece o desenvolvimento da via auditiva na mesma cronologia, porém, este estudo avaliou todas as crianças implantadas no serviço escolhido como lócus da pesquisa, onde a implantação de crianças acima dessa idade ainda era realidade.

O EDAL-1 (Quadro 1) é um teste composto por 20 questões fechadas, que permitem uma análise quantitativa das respostas. $\mathrm{O}$ instrumento deve ser aplicado aos pais/responsáveis pelas crianças sob avaliação. As questões 1a, 2a, 3a, 4a, e 6a são destinadas a crianças ouvintes e as questões $1 b, 2 b, 3 b, 4 b$, $5 \mathrm{~b}$ e $6 \mathrm{~b}$ são destinadas a crianças usuárias de prótese auditiva. A resposta à questão pode ser positiva (vale 5 pontos), ou negativa (vale zero), com exceção da questão $2 \mathrm{a}$, que foi invertida, porque a sua resposta, sendo positiva, tornaria-se um ponto negativo na avaliação. Ao final, somam-se as respostas positivas e obtém-se o escore total da criança, que pode variar de 0 a 100.

As respostas obtidas em cada grupo foram tabuladas e analisadas. Foram criadas categorias de idade auditiva de três em três meses, para estabelecimento de médias descritivas.

Para fins de análise estatística, as categorias etárias foram agrupadas em 0 a 6 meses, 6 meses e 1 dia a 15 meses e 15 meses e 1 dia a 24 meses. A comparação das médias obtidas entre as crianças foi realizada por meio do teste t de Student, com nível de significância de 0,05 . 


\section{RESULTADOS}

As 92 crianças do GC apresentaram resultados audiológicos normais na avaliação médica, na timpanometria (curvas tipo A e reflexo acústico presente) e na audiometria comportamental. O grupo foi composto por 43 (47\%) meninas e 49 (53\%) meninos, com idade cronológica variando de 0 a 2 anos de idade.

As 49 crianças do GC possuíam perda auditiva profunda e utilizavam IC unilateral. Este grupo continha 25 (51\%) meninas e $24(49 \%)$ meninos, com idade cronológica variando de 1 a 8 anos, porém, com idade auditiva entre 0 e 2 anos. Todas eram usuárias de prótese auditiva, sem benefício comprovado antes de receberem o IC. A média de idade para implantação foi de 3 anos e 8 meses.
Quando comparados os resultados obtidos por meio do teste $t$ de Student, com nível de significância de 0,05 (5\%), verificou-se diferença significativa entre as médias, para os dois grupos, nas três faixas etárias. Portanto, as médias foram significativamente maiores no GC, ou seja, as crianças ouvintes tiveram melhor desempenho no EDAL-1 do que as crianças implantadas com o mesmo tempo de idade auditiva.

Os resultados obtidos no EDAL-1, para cada faixa etária estabelecida, estão descritos na Tabela 1 e a comparação dos resultados entre GC e GE, apresentada na Tabela 2.

A partir das médias descritivas obtidas para os dois grupos avaliados, foi possível desenhar a curva de desempenho no teste, conforme demonstrado na Figura 1.

Quadro 1. Escala Brasileira de Desenvolvimento Infantil de Audição e Linguagem - 1

\begin{tabular}{|c|c|c|c|}
\hline Questão & Comportamento & Sim & Não \\
\hline 1 & $\begin{array}{l}\text { a) Você acha que o seu filho escuta? } \\
\text { b) A adaptação da criança ao dispositivo foi positiva? }\end{array}$ & & \\
\hline 2 & $\begin{array}{l}\text { a) Ele já teve problemas de ouvido? Quais? } \\
\text { b) Ela usa o dispositivo + de } 6 \text { horas por dia? }\end{array}$ & & \\
\hline 3 & $\begin{array}{l}\text { a) Ele gosta de ouvir música ou TV? } \\
\text { b) Ela manipula seu dispositivo? }\end{array}$ & & \\
\hline 4 & $\begin{array}{l}\text { a) Ele gosta de brinquedos barulhentos? } \\
\text { b) Seu comportamento muda quando está com o aparelho? }\end{array}$ & & \\
\hline 5 & $\begin{array}{l}\text { a) Ele reage a sons fortes? } \\
\text { b) Emite mais sons vocálicos quando põe o aparelho? }\end{array}$ & & \\
\hline 6 & \begin{tabular}{|l|} 
a. Ele acorda com barulhos? \\
b. Ela fica perturbada quando o dispositivo não funciona?
\end{tabular} & & \\
\hline 7 & Ele responde quando o chamam pelo nome, em silêncio? & & \\
\hline 8 & Ele responde quando chamam pelo nome, no ruído? & & \\
\hline 9 & Percebe sons ambientais do dia a dia? Quais? & & \\
\hline 10 & Vocaliza durante as interações comunicativas? & & \\
\hline 11 & Usa fala/vocalizações para atrair a atenção dos outros? Quais? & & \\
\hline 12 & As vocalizações variam de acordo com a situação? & & \\
\hline 13 & Tenta imitar sons, palavras ou vocalizações? Quais? & & \\
\hline 14 & Balança o corpo quando escuta música? & & \\
\hline 15 & Identifica diferentes vozes? & & \\
\hline 16 & Discrimina diferentes sons: voz, brinquedos, música? & & \\
\hline 17 & Responde a questionamentos simples sem apoio gestual? & & \\
\hline 18 & Fala palavras isoladas? Quais? & & \\
\hline 19 & Seu vocabulário vem ampliando? & & \\
\hline 20 & Fala duas palavras encadeadas? Quais? & & \\
\hline OTAL & Quantidade de Sim X 5 = & & \\
\hline
\end{tabular}

Fonte: Ribas e Kochen(7)

Legenda: a) questões a serem aplicadas em crianças normo-ouvintes; b) questões a serem aplicadas às crianças surdas implantadas

Tabela 1. Médias descritivas dos resultados da Escala Brasileira de Desenvolvimento Infantil de Audição e Linguagem - 1

\begin{tabular}{|c|c|c|c|c|c|c|}
\hline \multirow{2}{*}{$\begin{array}{c}\text { Idade } \\
\text { auditiva (em meses) }\end{array}$} & \multicolumn{3}{|c|}{ GC } & \multicolumn{3}{|c|}{ GE } \\
\hline & Média & Desvio padrão & Mediana & Média & Desvio padrão & Mediana \\
\hline $0-3$ & 34,23 & 8,12 & 30 & 29,28 & 12,53 & 25 \\
\hline $3,1-6$ & 54,68 & 8,65 & 55 & 43,88 & 17 & 50 \\
\hline $6,1-9$ & 73 & 10,59 & 75 & 42,14 & 14,23 & 45 \\
\hline $9,1-12$ & 82,50 & 10,34 & 80 & 44,54 & 12,93 & 45 \\
\hline $12,1-15$ & 87 & 7,52 & 85 & 61 & 21,95 & 55 \\
\hline $15,1-18$ & 91 & 5,16 & 90 & 66,50 & 22,97 & 65 \\
\hline $18,1-21$ & 92,50 & 6,34 & 95 & 72,85 & 21,18 & 70 \\
\hline $21,1-24$ & 95,83 & 5,06 & 95 & 82,77 & 17,25 & 90 \\
\hline
\end{tabular}

Legenda: GC = Grupo Controle; GE = Grupo Experimental 
Tabela 2. Comparação entre os resultados do grupo experimental e do grupo controle $(n=141)$

\begin{tabular}{|c|c|c|c|c|c|c|c|}
\hline \multirow{2}{*}{$\begin{array}{l}\text { Idade auditiva } \\
\text { (em meses) }\end{array}$} & \multicolumn{3}{|c|}{ Grupo Controle } & \multicolumn{3}{|c|}{ Grupo Estudo } & \multirow{2}{*}{ Valor de } \\
\hline & $\mathbf{n}$ & Média & Desvio padrão & $\mathrm{n}$ & Média & Desvio padrão & \\
\hline $0-6$ & 29 & 45,51 & 8,41 & 32 & 37,49 & 15,4 & 0,0070 \\
\hline $6,1-15$ & 30 & 80,83 & 9,48 & 35 & 48,28 & 16,03 & 0,0000 \\
\hline $15,1-24$ & 33 & 93,36 & 5,48 & 35 & 76,14 & 19,67 & 0,0000 \\
\hline
\end{tabular}

Obs.: Para possibilitar a aplicação do teste, foram consideradas apenas três faixas etárias, conforme mostra a tabela. Análise: Por meio do teste t de Student, com nível de significância de 0,05 (5\%), verificou-se diferença significativa entre as médias, para os dois grupos, nas três faixas etárias

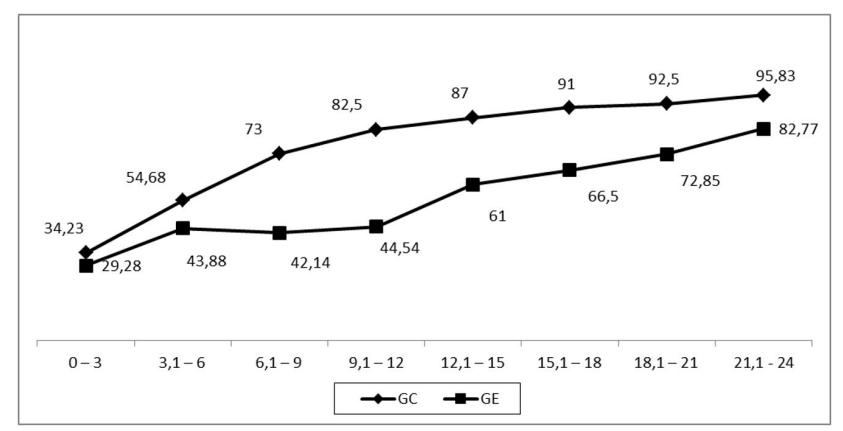

Figura 1. Curva de respostas considerando as médias por faixa de idade auditiva

\section{DISCUSSÃO}

Os dois grupos pesquisados apresentaram características homogêneas, em relação ao sexo e à idade auditiva, que esteve entre zero e dois anos. Por idade auditiva entende-se o tempo de estimulação auditiva que uma pessoa surda possui, após a adaptação do dispositivo eletrônico. Para as crianças normo-ouvintes, a idade cronológica é igual à idade auditiva ${ }^{(8)}$.

As crianças do GC tinham audição normal. A audição dentro da normalidade é fundamental nos primeiros anos de vida, que são considerados os mais importantes para o desenvolvimento das habilidades auditivas e de linguagem oral. É nesse período que ocorre a maturação do sistema nervoso, com maior crescimento cerebral e formação de novas conexões neuronais ${ }^{(9)}$. É na fase que vai de 0 aos 2 anos, conhecida como período pré-linguístico, que o bebê estabelece as bases da comunicação com os que o rodeiam. Pode-se dizer que é nesta fase que a criança aprende a escutar e a percepção dos sons da fala é o primeiro passo na compreensão da linguagem oral ${ }^{(10)}$.

As crianças do GE tinham perda auditiva profunda, incapacitante. A falta da audição nos primeiros anos de vida da criança compromete o seu desenvolvimento linguístico e cognitivo e gera impacto importante para o resto da vida. Neste sentido, diagnosticar a surdez e iniciar a reabilitação auditiva o mais cedo possível é fundamental ${ }^{(11)}$. Neste grupo, a média de idade em que as crianças receberam o IC foi de 3 anos e 8 meses, ou seja, atrasadas, em relação ao período pré-linguístico ${ }^{(10)}$. Pesquisas demonstraram que, quanto mais cedo o cérebro receber sons com significado, maiores condições as crianças terão de produzir bons resultados, devido à plasticidade funcional do sistema nervoso central e da diminuição da privação sensorial ${ }^{(8)}$.

Tanto no GC, quanto no GE, os resultados do EDAL 1 mostraram-se ascendentes (Figura 1), porém, as médias por faixa etária das crianças ouvintes foram sempre significativamente superiores às das crianças implantadas, apesar de a idade auditiva ser a mesma. Tal achado revela que a privação sensorial causa prejuízos ao desenvolvimento das habilidades auditivas e de linguagem. Apesar disso, se considerarmos o desvio padrão descrito na Tabela 1, é possível verificar que muitas crianças do GE alcançaram os resultados do GC. Tal dado reforça a necessidade de constante avaliação e acompanhamento de casos de crianças usuárias de $\mathrm{IC}^{(12-15)}$, com a finalidade de mapear as evoluções e oferecer subsídios para a conduta terapêutica.

A busca por melhores formas de avaliar a linguagem oral das crianças que utilizam IC é incessante e estimula estudiosos e pesquisadores da área, provavelmente em função das necessidades locais que cada serviço de saúde auditiva vivencia. Revisão sistemática $^{(15)}$ realizada há pouco tempo sobre o tema procurou examinar os instrumentos disponíveis. Os autores pesquisaram em três bancos de dados diferentes, utilizando seis descritores também diferentes, para selecionar artigos publicados de 2004 a 2009. Localizaram 47 artigos e perceberam que os estudos incluídos na revisão sistemática apresentaram metodologias variadas e baixos níveis de evidência, com maior concentração de instrumentos que avaliavam o idioma receptivo e expressivo, enfatizando a pesquisa do vocabulário e questionários da criança. Apontaram para o fato de que habilidades linguísticas (morfosintáticas, semânticas e narrativas/pragmáticas) importantes na estruturação de fala e linguagem para a eficácia do discurso da criança não estavam sendo focadas. Neste sentido, é importante esclarecer que o EDAL-1 é a primeira parte de um teste amplo, que se baseia na resposta dos pais sobre a evolução da audição e da linguagem das crianças implantadas e foca os primeiros meses de reabilitação com IC.

Considerando-se que o EDAL-1 é um protocolo desenvolvido por fonoaudiólogas para a realidade dos ambulatórios de saúde auditiva do SUS ${ }^{(7)}$, é possível afirmar que o instrumento se mostrou efetivo no que se refere ao acompanhamento das crianças recém-implantadas.

A partir dos dados coletados no EDAL-1, foi possível traçar uma linha de desenvolvimento de cada criança implantada, comparando-se os achados registrados em cada uma das sessões de acompanhamento, e visualizar os avanços realizados desde o dia da ativação. Essa comparação permite monitorar o desempenho auditivo e de linguagem em crianças implantadas e, assim, fornecer subsídios para a terapia fonoaudiológica ${ }^{(13,15)}$.

Também é possível comparar os resultados das crianças implantadas com os de crianças ouvintes e, assim, estabelecer metas para as famílias e fonoaudiólogos terapeutas, em busca de melhores respostas com o uso do dispositivo.

Apesar de ser um instrumento de medida indireta, que se baseia nas respostas de um observador, há que se pensar que a comunicação é dependente da interação bidirecional entre pais e filhos e deve ser considerada na avaliação de crianças pequenas em processo de reabilitação. 


\section{CONCLUSÃO}

O desempenho auditivo e de linguagem oral em crianças surdas, usuárias de implante coclear, foi caracterizado pelo EDAL-1 e se mostrou em crescimento, conforme o aumento do tempo de estimulação auditiva, fato também observado nas crianças ouvintes. Apesar disso, os resultados das crianças ouvintes foram melhores que os das crianças surdas implantadas.

\section{REFERÊNCIAS}

1. Hyppolito MA, Bento RF. Directions of the bilateral cochlear implant in Brazil. Braz J Otorhinolaryngol. 2012;78(1):2-3. http://dx.doi. org/10.1590/S1808-86942012000100001. PMid:22392231.

2. Costa OA, Bevilacqua MC, Amantini RCB. Considerações sobre o implante coclear em crianças. In: Bevilacqua MC, Moret ALM, organizadores. Deficiência auditiva. Conversando com familiares e profissionais de saúde. São José dos Campos: Pulso; 2005. p. 123-37.

3. Grieco-calub TM, Saffran JR, Litovsky RY. Spoken word recognition in toddlers who use cochlear implants. J Speech Lang Hear Res. 2009;52(6):1390-400. http://dx.doi.org/10.1044/1092-4388(2009/080154). PMid:19951921.

4. Gilley PM, Sharma A, Dorman MF. Cortical reorganization in children with cochlear implants. Brain Res. 2008;1239:56-65. http://dx.doi. org/10.1016/j.brainres.2008.08.026. PMid:18775684.

5. Brasil. Ministério da Saúde. Portaria $n^{\circ} 2.776$, de 18 de dezembro de 2014. Aprova diretrizes gerais, amplia e incorpora procedimentos para a Atenção Especializada às Pessoas com Deficiência Auditiva no Sistema Único de Saúde (SUS). Brasília, DF: Ministério da Saúde; 2014.

6. Ribas A, Patruni T, Ataide AL, Pereira R, Moretti CAM, Vassoler $\mathrm{T}$ et al. Epidemiological and demographic profile of patients at a pediatric hospital in southern Brazil. In: Proceedings of the European Symposiun on Pediatric Cochlear Implants; 2015 Dec; Toulouse. Toulouse: ESPCI; 2015. p. 145.
7. Ribas A, Kochen AP. Brazilian scale of hearing and language development in children (EDAL-1) with cochlear implant and less than two years of hearing AGE. Int Tinnitus J. 2016;20(1):7-10. http:// dx.doi.org/10.5935/0946-5448.20160002. PMid:27488987.

8. Hilgenberg AMS, Cardoso CC, Caldas FF, Tschiedel RS, Deperon TM, Bahmad F Jr. Hearing rehabilitation in cerebral palsy: development of language and hearing after cochlear implantation. Braz J Otorhinolaryngol. 2015;81(3):240-7. http://dx.doi.org/10.1016/j.bjorl.2014.10.002. PMid:25458256.

9. Friederici AD. The neural basis of language development and its impairment. Neuron. 2006;52(6):941-52. http://dx.doi.org/10.1016/j. neuron.2006.12.002. PMid:17178399.

10. Pedroso FS, Rotta NT, Danesi MC, Avila LN, Savio CB. Evolução das manifestações pré-linguísticas em crianças normais no primeiro ano de vida. Rev Soc Bras Fonoaudiol. 2009;14(1):22-5. http://dx.doi. org/10.1590/S1516-80342009000100006.

11. Bellis TJ.Assessment and management of central auditory processing disorders in the educational setting. 2nd ed. San Diego: Singular; 2002.

12. Fortunato CAU, Bevilacqua MC, Costa MPR. Análise comparativa da linguagem oral de crianças ouvintes e surdas usuárias de implante coclear. Rev CEFAC. 2009;11(4):662-72. http://dx.doi.org/10.1590/ S1516-18462009000800015.

13. Niparko JK, Tobey EA, Thal DJ, Eisenberg LS, Wang NY, Quittner AI, Fink NE. Spoken language development in children following cochlear implantation. JAMA. 2010;303(15):1498-506. http://dx.doi. org/10.1001/jama.2010.451. PMid:20407059.

14. Melo TM, Bevilacqua MC, Costa OA. Percepção da fala de usuários de implante coclear com a estratégia HiRes 120: revisão sistemática. Rev Bras Otorrinolaringol. 2012;78(3):129-33. http://dx.doi.org/10.1590/ S1808-86942012000300021.

15. Silva MP, Comerlatto AA Jr, Bevilacqua MC, Lopes-Herrera SA. Instruments to assess the oral language of children fitted with a cochlear implant: a systematic review. J Appl Oral Sci. 2011;19(6):549-53. http:// dx.doi.org/10.1590/S1678-77572011000600002. PMid:22230986. 